Modern Physics Letters A

(C) World Scientific Publishing Company

\title{
Casimir effect with a helix torus boundary condition
}

\author{
XIANG-HUA ZHAI* XIN-ZHOU LI ${ }^{\dagger}$ and CHAO-JUN FENG ${ }^{\ddagger}$ \\ Shanghai United Center for Astrophysics (SUCA), Shanghai Normal University, 100 Guilin \\ Road, 100 Guilin Road, Shanghai 200234, China \\ Received (Day Month Year) \\ Revised (Day Month Year)
}

\begin{abstract}
We use the generalized Chowla-Selberg formula to consider the Casimir effect of a scalar field with a helix torus boundary condition in the flat $(D+1)$-dimensional spacetime. We obtain the exact results of the Casimir energy density and pressure for any $D$ for both massless and massive scalar fields. The numerical calculation indicates that once the topology of spacetime is fixed, the ratio of the sizes of the helix will be a decisive factor. There is a critical value $r_{\text {crit }}$ of the ratio $r$ of the lengths at which the pressure vanishes. The pressure changes from negative to positive as the ratio $r$ passes through $r_{\text {crit }}$ increasingly. In the massive case, we find the pressure tends to the result of massless field when the mass approaches zero. Furthermore, there is another critical ratio of the lengths $r_{c r i t}^{\prime}$ and the pressure is independent of the mass at $r=r_{c r i t}^{\prime}$ in the $D=3$ case.

Keywords: Casimir effect; zeta function; boundary condition; Chowla-Selberg formula.
\end{abstract}

PACS Nos.: 02.30.Gp; 11.10.-z

\section{Introduction}

Casimir's calculation of the force between two neutral, parallel conducting plates originally inspired much theoretical interest as macroscopic manifestation of quantum fluctuation of the field in vacuum. However, the Casimir effect arises not only in the presence of material boundaries, but also in spaces with non-Euclidean topology 1 . The simplest example of the Casimir effect of topological origin is the scalar field on a flat manifold with topology of a circle $S^{1}$. The topology of $S^{1}$ causes the periodicity condition $\phi(t, 0)=\phi(t, C)$ for a Hermitian scale field $\phi(t, x)$, where $C$ is the circumference of $S^{1}$, imposed on the wave function which is of the same kind as those due to boundary and resulting in an attractive Casimir force. Similarly, the antiperiodic conditions can be drawn on a Möbius strip and bring about the repulsive Casimir force as a result. Recently, the topology of the helix boundary conditions is investigated in ref. ${ }^{2}$. We find that the Casimir effect is very

\footnotetext{
*zhaixh@shnu.edu.cn

${ }^{\dagger} \mathrm{kychz@shnu.edu.cn}$

$\ddagger_{\text {fengcj@shnu.edu.cn }}$
} 
much like the effect on a spring that obeys the Hooke's law in mechanics. However, in this case, the pressure comes from a quantum effect, so we would like to call this structure a quantum spring. The pressurre is negative in both massless and massive scalar cases for this structure 3 .

It is worth noting that the concept of quotient topology is very useful for concrete application. We consider a surjective mapping $f$ from a topological space $X$ onto a set $Y$. The quotient topology on $Y$ with respect to $f$ is given in ${ }^{4}$. Surjective mapping can be easily obtained when we use the equivalence classes of some equivalence relation $\sim$. Thus, we let $X / \sim$ denote the set of equivalence classes and define $f: X \rightarrow X / \sim$ by $f(x)=[x]$ the equivalence class containing $x . X / \sim$ with the quotient topology is called to be obtained from $X$ by topological identification. For example, if we take the rectangle $Y=\left\{\left(x^{1}, x^{2}\right) ; 0 \leq x^{1} \leq a, 0 \leq x^{2} \leq h\right\}$ in $\mathbb{R}^{2}$ with the induced topology and define an equivalence relation $\sim$ on $Y$ by $\left(x^{1}, x^{2}\right) \sim\left(x^{1 \prime}, x^{2 \prime}\right) \Leftrightarrow\left(x^{1}, x^{2}\right)=\left(x^{\prime 1}, x^{\prime 2}\right)$ or $\left\{x^{1}, x^{\prime 1}\right\}=\{0, a\}$ and $x^{2}=x^{\prime 2}$, then $Y / \sim$ with the quotient topology is homomorphic to the cylinder $C=\{(x, y, z) \in$ $\left.\mathbb{R}^{3} ; x^{2}+y^{2}=\left(\frac{a}{2 \pi}\right)^{2},|z| \leq 1\right\}$. The boundary condition $\phi\left(t, 0, x^{2}\right)=\phi\left(t, a, x^{2}\right)$ can be drawn on the topology of a cylinder. Similarly, we define another equivalence relation $\sim$ on $Y$ by $\left(x^{1}, x^{2}\right) \sim\left(x^{\prime 1}, x^{\prime 2}\right) \Leftrightarrow\left(x^{1}, x^{2}\right)=\left(x^{\prime 1}, x^{\prime 2}\right)$ or $x^{1}=0, x^{1}=a, x^{2}=x^{\prime 2}$ and $x^{1}=x^{11}, x^{2}=0, x^{\prime 2}=h$, then $Y / \sim$ with the quotient topology is homomorphic to a torus $T=\left\{(x, y, z) \in \mathbb{R}^{3} ;\left(\frac{a}{2 \pi}-\sqrt{x^{2}+y^{2}}\right)^{2}+z^{2}=\left(\frac{h}{2 \pi}\right)^{2}, 0 \leq h \leq a\right\}$. The boundary conditions $\phi\left(t, 0, x^{2}\right)=\phi\left(t, a, x^{2}\right)$ and $\phi\left(t, x^{1}, 0\right)=\phi\left(t, x^{1}, h\right)$ can be drawn on the topology of the torus. In this paper, we will consider the helix torus topology using the concept of quotient topology.

The $\zeta$-function regularization procedure is a powerful and elegant technique for the Casimir effect 5 . The generalized $\zeta$-function has many interesting applications, e.g., in the piecewise string 617. Similar analysis has been applied to rectangular cavity $\underline{810}$, noncommutative spacetime ${ }^{11}$, p-branes 12 or pistons 13 17. Casimir effect for a fractional boundary condition is of interest in considering, for example, the finite temperature Casimir effect for a scalar field with fractional Neumann conditions 18 , while the repulsive force from fractional boundary conditions has been studied 19. The Chowla-Selberg formula of the $\zeta$-function has been applied to quantize the Wheelar-DeWitt equation 20 and recently to calculate the Casimir energies of cylinders 21 .

In this paper, we consider the Casimir effect of a scalar field with a helix torus boundary condition in the flat $(D+1)$-dimensional spacetime. The Chowla-Selberg formula and its generalization are used to regularize the Casimir energy density. We obtain the exact results of the Casimir energy and pressure for any $D$ for both massless and massive scalar fields. The numerical calculation indicates that once the topology of spacetime is fixed, the ratio of the sizes of the helix will be a decisive factor. In massless case, there is a critical value $r_{\text {crit }}$ of the ratio $r$ of the lengths at which the pressure vanishes. The pressure changes from negative to positive as the ratio $r$ passes through $r_{c r i t}$ increasingly. In massive case, we compare the pressure of 
massive field with massless one and find the pressure tends to the result of massless field when the mass approaches zero. Furthermore, we find there is also a critical ratio $r_{c r i t}(\mu)$ at which the pressure vanishes. At the same time, there is another critical ratio of the lengths $r_{c r i t}^{\prime}$ and the pressure is independent of the mass at $r=r_{\text {crit }}^{\prime}$ in the $D=3$ case. The outline of this paper is as follows. In Sec. 2 we introduce a specified helix topology for the flat spacetime. In Sec. 3 we consider the evaluation of the Casimir effect for massless and massive cases. The results are summarized in Sec. 4.

\section{Helix torus topology}

The Casimir effect arises not only in the presence of material boundaries, but also in spaces with non-Euclidean topology. First, we consider a specified 2-dimensional helix topology which may be obtained by identifying some or whole in a series of rectangles as a pedagogical discussion. The whole situation concerning the helix boundary conditions in this and similar cases is discussed in $2 \mid 3$ in great detail. In Fig. 1(a), the cylinder is obtained by identifying some of the boundary points of a rectangle, in which we intend to identify the two edges. This is often indicated by labeling the two points of edges with the same letter, such as A, B. Similarly, we may obtain a torus (see Fig. 1(b)). Furthermore, a helix torus topology can occur by identifying both distinct rectangles and the boundary points at the same rectangle(see Fig. 1(c)).

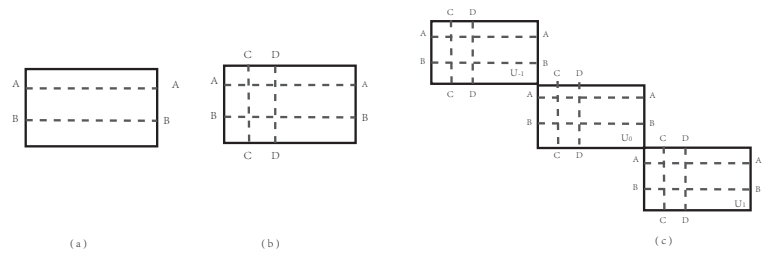

Fig. 1. There are many topologies that may be obtained by identifying various boundary points of a rectangle or many rectangles. (a) A cylindrical topology: we may equally well picture the cylinder as being the topological space obtained by identifying the two edges. This is indicated by labeling the two points of edges with the same letter. (b) A torus topology: we may equally well picture the torus as being the topological space obtained by identifying the two pairs of edges in a rectangle. In other words, a torus topology occurs if we join two side of rectangle, then join the other perpendicular direction as well. (c) A helix torus topology: A helix topology may be obtained by identifying some or whole in a series of rectangles, in contrast to the case of cylindroid or torus. A helix torus topology may be obtained by identifying both distinct rectangles and the boundary points at the same rectangle. In this figure, we show 3 rectangles which are denoted $U_{i}(i=-1,0,1)$ respectively. Here, $U_{-1}=\left\{x^{1} \mathbb{e}_{1}+x^{2} \mathbb{e}_{2} \mid-a \leq x^{1} \leq 0, h \leq x^{2} \leq 2 h\right\}, U_{0}=$ $\left\{x^{1} \mathbb{e}_{1}+x^{2} \mathbb{e}_{2} \mid 0 \leq x^{1} \leq a, 0 \leq x^{2} \leq h\right\}$ and $U_{1}=\left\{x^{1} \mathfrak{e}_{1}+x^{2} \mathbb{e}_{2} \mid a \leq x^{1} \leq 2 a,-h \leq x^{2} \leq 0\right\}$, and every $U_{i}$ has torus topology.

Before we consider a helix torus topology in the flat $(D+1)$-dimensional spacetime $\mathcal{M}^{D+1}$ using the concept of quotient topology, we have to discuss the lattices. 
A lattice $\Lambda$ is defined as a set of points

$$
\Lambda=\left\{\sum_{i=0}^{D} n_{i} \mathbb{e}_{i} \mid n_{i} \in \mathcal{Z}\right\},
$$

where $\left\{\mathbb{e}_{i}\right\}$ is a set of basis vectors of $\mathcal{M}^{D+1}$. In terms of the components $v^{i}$ of vectors $\mathbb{V} \in \mathcal{M}^{D+1}$, we define the inner products as

$$
\mathbb{V} \cdot \mathbb{W}=\epsilon(a) v^{i} w^{j} \delta_{i j}
$$

with $\epsilon(a)=1$ for $i=0, \epsilon(a)=-1$ for otherwise. In the $x^{1}-x^{2}$ plane, the sublattice $\Lambda^{\prime \prime} \subset \Lambda^{\prime} \subset \Lambda$ are

$$
\Lambda^{\prime}=\left\{n_{3} \mathbb{E}_{1}+n_{2} \mathbb{E}_{2} \mid n_{2}, n_{3} \in \mathcal{Z}\right\},
$$

and

$$
\Lambda^{\prime \prime}=\left\{n_{1}\left(\mathbb{e}_{1}-\mathbb{e}_{2}\right) \mid n_{1} \in \mathcal{Z}\right\} .
$$

The unit cell is the set of points

$$
\begin{aligned}
C_{0}=\{\mathbf{X}= & \sum_{i=0}^{D} x^{i} \mathbb{e}_{i} \mid 0 \leq x^{1} \leq a,-h \leq x^{2} \leq 0,\left(0, x^{2}\right) \Leftrightarrow\left(a, x^{2}\right) \\
& \left.\operatorname{and}\left(x^{1}, 0\right) \Leftrightarrow\left(x^{1},-h\right) ;-\infty<x^{0}<\infty,-\frac{L}{2} \leq x^{T} \leq \frac{L}{2}\right\},
\end{aligned}
$$

where $T=3, \cdots, D$ and $\Leftrightarrow$ is a symbol of identity relation.

Next, we choose topological space $\mathbf{X}$ as $\mathbf{X}=\bigcup_{\mathfrak{u} \in \Lambda^{\prime \prime}}\left\{C_{0}+\mathbb{u}\right\}$ in $\mathcal{M}^{D+1}$ with the induced topology and define an equivalence relation $\sim$ on $\mathbf{X}$ by $\left(x^{1}, x^{2}\right) \sim$ $\left(x^{1}-a, x^{2}+h\right)$, then $\mathbf{X} / \sim$ with the quotient topology is a new type topology which can be called a helix torus topology. This topology causes the helix boundary condition for a Hermitian scalar field

$$
\phi\left(t, x^{1}+a, x^{2}, x^{T}\right)=\phi\left(t, x^{1}, x^{2}+h, x^{T}\right),
$$

and the periodicity boundary conditions

$$
\phi\left(t, x^{1}, 0, x^{T}\right)=\phi\left(t, x^{1}, h, x^{T}\right),
$$

and

$$
\phi\left(t,-a, x^{2}, x^{T}\right)=\phi\left(t, 0, x^{2}, x^{T}\right) .
$$

It is worth noting that Eq.(8) can be derived from Eqs.(6) and (7), or Eq.(77) derived from Eqs.(6) and (8). Therefore, we need only one of the two periodicity boundary conditions. 


\section{Evaluation of the Casimir effect}

\subsection{The Casimir energy density}

In calculations on the Casimir effect, extensive use is made of eigenfunctions and eigenvalues of the corresponding field equation. A Hermitian scalar field $\phi\left(t, x^{\alpha}, x^{T}\right)$ defined in the $(D+1)$-dimensional flat spacetime satisfies the Klein-Gordon equation:

$$
\left(\partial_{t}^{2}-\partial_{i}^{2}+\mu^{2}\right) \phi\left(t, x^{\alpha}, x^{T}\right)=0,
$$

where $i=1, \cdots, D ; \alpha=1,2 ; T=3, \cdots, D$ and $\mu$ is the mass of the scalar field. Under the boundary condition (6) and (17), the modes of the field are then

$$
\phi_{n}\left(t, x^{\alpha}, x^{T}\right)=\mathcal{N} e^{-i \omega_{n} t+i k_{x} x+i k_{z} z+i k_{T} x^{T}},
$$

where $\mathcal{N}$ is a normalization factor and $x^{1}=x, x^{2}=z$, and we have

$$
\omega_{n}^{2}=k_{T}^{2}+k_{x}^{2}+\left(-\frac{2 \pi n_{1}}{h}+\frac{k_{x}}{h} a\right)^{2}+\mu^{2}=k_{T}^{2}+k_{z}^{2}+\left(\frac{2 \pi n_{1}}{a}+\frac{k_{z}}{a} h\right)^{2}+\mu^{2} .
$$

Here, $k_{x}$ and $k_{z}$ satisfy

$$
\begin{gathered}
a k_{x}-h k_{z}=2 n_{1} \pi, \\
k_{z}=\frac{2 \pi n_{2}}{h}, k_{x}=\frac{2 \pi n_{3}}{a},
\end{gathered}
$$

where $n_{2}, n_{3}=0, \pm 1, \pm 2, \cdots$ and the constraint condition $n_{1}=n_{3}-n_{2}$. In the ground state (vacuum), each of these modes contributes an energy of $\omega_{n} / 2$. The energy density of the field is thus given by

$$
\varepsilon^{D}=\frac{1}{2 a} \int \frac{d^{D-2} k_{T}}{(2 \pi)^{D-2}} \sum_{n_{1}, n_{2}=-\infty}^{\infty \prime} \sqrt{k_{T}^{2}+\left(\frac{2 \pi n_{2}}{h}\right)^{2}+\left(\frac{2 \pi n_{1}}{a}+\frac{2 \pi n_{2}}{a}\right)^{2}+\mu^{2}},
$$

where we have assumed $a \neq 0$ and $h \neq 0$ without losing generalities and the prime on the summation means that $\left(n_{1}, n_{2}\right)=(0,0)$ have to be omitted. Remember that there are no material boundaries here and the Casimir effect arises owing to the nontrivial topology. This allows us to define not only the separation-dependent global energy but also the energy density as above.

Using the mathematical identity

$$
\int_{-\infty}^{\infty} f(u) d^{D-2} u=\frac{2 \pi^{\frac{D-2}{2}}}{\Gamma\left(\frac{D-2}{2}\right)} \int_{0}^{\infty} u^{D-3} f(u) d u
$$

Eq. (14) can be reduced to

$$
\begin{aligned}
\varepsilon^{D}= & -\frac{1}{2 a} \pi^{\frac{D-1}{2}} \Gamma\left(-\frac{D-1}{2}\right) \\
& \times \sum_{n_{1}, n_{2}=-\infty}^{\infty \prime}\left[\frac{n_{1}^{2}}{a^{2}}+\frac{2 n_{1} n_{2}}{a^{2}}+\left(\frac{1}{a^{2}}+\frac{1}{h^{2}}\right) n_{2}^{2}+\left(\frac{\mu}{2 \pi}\right)^{2}\right]^{\frac{D-1}{2}} .
\end{aligned}
$$


On the other hand, the generalized Chowla-Selberg formula was given firstly by Elizalde et. al. 5 and was derived in detail recently by Abalo et. al. 21

$$
\begin{aligned}
S & =\Gamma(s) \sum_{m, n=-\infty}^{\infty \prime}\left(\alpha m^{2}+\beta m n+\gamma n^{2}+\delta\right)^{-s} \\
& =2 \Gamma(s) \zeta\left(s, \frac{\delta}{\alpha}\right) \alpha^{-s}+\frac{2^{2 s} \sqrt{\pi} \alpha^{s-1}}{\Delta^{s-\frac{1}{2}}} \Gamma\left(s-\frac{1}{2}\right) \zeta\left(s-\frac{1}{2}, \frac{4 \alpha \delta}{\Delta}\right) \\
& +4(2 \pi)^{s} \sqrt{\frac{2}{\alpha}} \sum_{n=1}^{\infty} n^{s-\frac{1}{2}} \cos \left(\frac{n \pi \beta}{\alpha}\right) \sum_{d \mid n} d^{1-2 s}\left(\Delta+\frac{4 \alpha \delta}{d^{2}}\right)^{\frac{1-2 s}{4}} \\
& \times K_{s-\frac{1}{2}}\left(\frac{\pi n}{\alpha} \sqrt{\Delta+\frac{4 \alpha \delta}{d^{2}}}\right) .
\end{aligned}
$$

where $\Delta=4 \alpha \gamma-\beta^{2}, d \mid n$ denotes the divisor of $n, k_{\nu}(z)$ is the modified Bessel function and $\zeta(s, p)$ is the Epstein-Hurwitz $\zeta$ function defined as

$$
\zeta(s, p) \equiv \sum_{n=1}^{\infty}\left(n^{2}+p\right)^{-s} .
$$

By using Eq.(17), the infinite summation in Eq.(16) can be regularized. In the following subsections, We discuss the massless and massive cases respectively.

\subsection{The massless case}

In the massless case $\mu=0$, using Eq. (17) with $\delta=0$ and the functional relation

$$
\pi^{-\frac{s}{2}} \Gamma\left(\frac{s}{2}\right) \zeta(s)=\pi^{-\frac{1-s}{2}} \Gamma\left(\frac{1-s}{2}\right) \zeta(1-s),
$$

one can rewrite the Casimir energy density of massless scalar field as

$$
\begin{aligned}
\varepsilon_{\mu=0}^{D}= & -\frac{\Gamma\left(\frac{D}{2}\right) \zeta(D)}{\pi^{\frac{D}{2}} a^{D}}-\frac{\Gamma\left(\frac{D+1}{2}\right) \zeta(D+1)}{\pi^{\frac{D+1}{2}} h^{D}} \\
& -\frac{4}{(a h)^{\frac{D}{2}}} \sum_{n_{1}, n_{2}=1}^{\infty}\left(\frac{n_{2}}{n_{1}}\right)^{\frac{D}{2}} K_{\frac{D}{2}}\left(\frac{2 \pi n_{1} n_{2} a}{h}\right),
\end{aligned}
$$

where

$$
\sum_{n_{1} n_{2}=1}^{\infty}\left(\frac{n_{1}}{n_{2}}\right)^{s-\frac{1}{2}}=\sum_{n=1}^{\infty} n^{s-\frac{1}{2}} \sum_{d \mid n} d^{1-2 s} .
$$

In the $D=3$ case, Eq.(20) is reduced to

$$
\varepsilon_{\mu=0}=-\frac{\zeta(3)}{2 \pi a^{3}}-\frac{\pi^{2}}{90 h^{3}}-\frac{4}{a^{\frac{3}{2}} h^{\frac{3}{2}}} \sum_{n_{1}, n_{2}=1}^{\infty}\left(\frac{n_{2}}{n_{1}}\right)^{\frac{3}{2}} K_{\frac{3}{2}}\left(\frac{2 \pi n_{1} n_{2} a}{h}\right) .
$$


From the thermodynamic relation, one can get the pressure on the $x^{1}$ direction as

$$
\begin{aligned}
P_{a, \mu=0} & =-\frac{\partial E_{\mu=0}}{\partial a} \\
& =-\frac{\zeta(3)}{\pi a^{3}}+\frac{\pi^{2}}{90 h^{3}}+\frac{2}{(a h)^{\frac{3}{2}}} \sum_{n_{1}, n_{2}=1}^{\infty}\left(\frac{n_{2}}{n_{1}}\right)^{\frac{3}{2}} K_{\frac{3}{2}}\left(\frac{2 \pi n_{1} n_{2} a}{h}\right) \\
& -\frac{8 \pi}{a^{\frac{1}{2}} h^{\frac{5}{2}}} \sum_{n_{1}, n_{2}=1}^{\infty}\left(\frac{n_{2}}{n_{1}}\right)^{\frac{1}{2}} n_{2}^{2} K_{\frac{5}{2}}\left(\frac{2 \pi n_{1} n_{2} a}{h}\right) .
\end{aligned}
$$

where $E_{\mu=0}=a \varepsilon_{\mu=0}$. It is worth noting that the pressure can also be obtained from the component of the energy-momentum tensor which has the physical meaning of a pressure.

By the numerical calculation, we have $P_{a, \mu=0}=0$ when $a=r_{\text {crit }} h$ and $r_{c r i t}=$ $1.52007606 \cdots$. It is obvious that $P_{a, \mu=0}$ is negative if $a<r_{c r i t} h$ and is positive if $a>r_{c r i t} h$ since the first and fourth terms are negative and the second and third terms are positive in Eq.(23). When $a \gg h$, we have $F_{a} \approx \frac{\pi^{2}}{90 h^{3}}$. Fig. 2 is the illustration of the behavior of the pressure on $x^{1}$ direction for $D=3$. The curves correspond to $h=1,2,3,4$ respectively. It is clearly seen that for a given $h$, the negative pressure become positive with $a$ increasing. Once topology of spacetime is fixed, the ratio of the sizes of the helix will be a decisive factor.

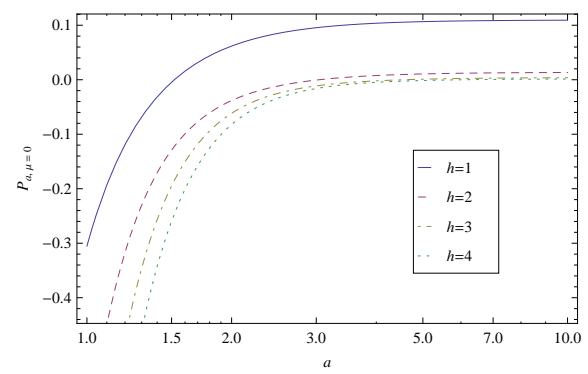

Fig. 2. The pressure $P_{a, \mu=0}$ on the $x^{1}$ direction for $D=3$ and $h=1,2,3,4$. It is clearly seen that the pressure $P_{a, \mu=0}$ is a monotonic function and vanishes at $a=r_{\text {crit }} h$. It changes from attractive to repulsive when passing through the critical ratio $r_{c r i t}$ increasingly and tends to $\frac{\pi^{2}}{90 h^{3}}$. 


\subsection{The massive case}

In the $\mu \neq 0$ case, using Eq. (17), we have

$$
\begin{aligned}
& \Gamma\left(-\frac{D+1}{2}\right) \sum_{n_{1}, n_{2}=-\infty}^{\infty \prime}\left[\frac{n_{1}^{2}}{a^{2}}+\frac{2 n_{1} n_{2}}{a^{2}}+\left(\frac{1}{a^{2}}+\frac{1}{h^{2}}\right) n_{2}^{2}+\left(\frac{\mu}{2 \pi}\right)^{2}\right]^{\frac{D-1}{2}} \\
= & 2 \Gamma\left(-\frac{D+1}{2}\right) \zeta\left(-\frac{D-1}{2}, \frac{a^{2} \mu^{2}}{4 \pi^{2}}\right) a^{1-D}+\frac{\sqrt{\pi} a^{D+1}}{(a h)^{D}} \Gamma\left(-\frac{D}{2}\right) \zeta\left(-\frac{D}{2}, \frac{\mu^{2} h^{2}}{4 \pi^{2}}\right) \\
+ & \frac{8 \pi^{\frac{1-D}{2}}}{a^{\frac{D}{2}-1} h^{\frac{D}{2}}} \sum_{n_{1}, n_{2}=1}^{\infty}\left(\frac{\sqrt{n_{2}^{2}+\left(\frac{\mu}{2 \pi}\right)^{2} h^{2}}}{n_{1}}\right)^{\frac{D}{2}} K_{\frac{D}{2}}\left(\frac{2 \pi a n_{1}}{h} \sqrt{n_{2}^{2}+\left(\frac{\mu}{2 \pi}\right)^{2} h^{2}}\right) .
\end{aligned}
$$

From the relation between the Epstein-Hurwitz $\zeta$ function and the Riemann $\zeta$ function $\zeta(s, 0)=\zeta(2 s)$ and Eq.(19), we obtain again the massless expression when $\mu \rightarrow 0$.

Using Eqs.(18) and (24), we have the global energy between the separation $a$ from Eq.(16) as

$$
\begin{aligned}
E^{D} & =\frac{1}{2} \pi^{\frac{D-1}{2}} \Gamma\left(\frac{1-D}{2}\right)\left(\frac{\mu}{2 \pi}\right)^{D-1}-\frac{1}{2} \pi^{\frac{D+1}{2}} \Gamma\left(-\frac{D+1}{2}\right)\left(\frac{\mu}{2 \pi}\right)^{D+1} a h \\
& -2\left(\frac{\mu a}{2 \pi}\right)^{\frac{D}{2}} a^{1-D} \sum_{n_{2}=1}^{\infty} n_{2}^{-\frac{D}{2}} K_{\frac{D}{2}}\left(n_{2} \mu a\right) \\
& -2\left(\frac{\mu h}{2 \pi}\right)^{\frac{D+1}{2}} h^{-D} a \sum_{n_{2}=1}^{\infty} n_{2}^{-\frac{D+1}{2}} K_{\frac{D+1}{2}}\left(n_{2} \mu h\right) \\
& -4 a^{1-\frac{D}{2}} h^{-\frac{D}{2}} \sum_{n_{1}, n_{2}=1}^{\infty}\left(\frac{\sqrt{n_{2}^{2}+\left(\frac{\mu}{2 \pi}\right)^{2} h^{2}}}{n_{1}}\right)^{\frac{D}{2}} K_{\frac{D}{2}}\left(\frac{2 \pi a n_{1}}{h} \sqrt{n_{2}^{2}+\left(\frac{\mu}{2 \pi}\right)^{2} h^{2}}\right) .
\end{aligned}
$$

Ambjørn and Wolfram 22 have treated the case of Dirichlet boundary condition for a massive scalar field. Following their methodology, we get the finite physically relevant energy. The first term in Eq.(25) gives a contribution to the total energy independent of $a$ and $h$, therefore it can be dropped. The second term in Eq. (25) corresponds to a constant energy density which can be canceled by addition of a constant to the Hamiltonian density. Therefore, the finite physically relevant energy 
is

$$
\begin{aligned}
E^{D}= & -2\left(\frac{\mu a}{2 \pi}\right)^{\frac{D}{2}} a^{1-D} \sum_{n_{2}=1}^{\infty} n_{2}^{-\frac{D}{2}} K_{\frac{D}{2}}\left(n_{2} \mu a\right) \\
& -2\left(\frac{\mu h}{2 \pi}\right)^{\frac{D+1}{2}} h^{-D} a \sum_{n_{2}=1}^{\infty} n_{2}^{-\frac{D+1}{2}} K_{\frac{D+1}{2}}\left(n_{2} \mu h\right) \\
& -4 a^{1-\frac{D}{2}} h^{-\frac{D}{2}} \sum_{n_{1}, n_{2}=1}^{\infty}\left(\frac{\sqrt{n_{2}^{2}+\left(\frac{\mu}{2 \pi}\right)^{2} h^{2}}}{n_{1}}\right)^{\frac{D}{2}} K_{\frac{D}{2}}\left(\frac{2 \pi a n_{1}}{h} \sqrt{n_{2}^{2}+\left(\frac{\mu}{2 \pi}\right)^{2} h^{2}}\right) .
\end{aligned}
$$

Using the expressions of the modified Bessel function

$$
K_{j+\frac{1}{2}}(z)=\sqrt{\frac{\pi}{2 z}} e^{-z} \sum_{k=0}^{j} \frac{(j+k) !}{k !(j-k) !(2 z)^{k}}
$$

and

$$
\begin{aligned}
K_{j}(z)= & \frac{1}{2} \sum_{k=0}^{j-1}(-1)^{k} \frac{(j-k-1) !}{k !\left(\frac{z}{2}\right)^{j-2 k}}+(-1)^{j+1} \sum_{k=0}^{\infty} \frac{\left(\frac{z}{2}\right)^{j+2 k}}{k !(j+k) !} \\
& \times\left[\ln \frac{3}{2}-\frac{1}{2} \psi(k+1)-\frac{1}{2} \psi(j+k+1)\right]
\end{aligned}
$$

where $\psi(k+1)=-c+\left(1+\frac{1}{2}+\cdots+\frac{1}{k}\right)$ and $c$ is the Euler's constant, we can calculate the Casimir energy for massive case. Especially, we have the physical Casimir energy for small mass $\mu$ from Eqs.(26)-(28) up to $\mu^{2}$ order,

$$
\begin{aligned}
E^{D}= & E_{\mu=0}^{D}+\frac{1}{4} a^{1-D} \pi^{-\frac{D}{2}} \Gamma\left(\frac{D-2}{2}\right) \zeta(D-2)\left(1-\delta_{D}^{3}\right)(\mu a)^{2} \\
& +\frac{1}{4} h^{-D} a \pi^{-\frac{D+1}{2}} \Gamma\left(\frac{D-1}{2}\right) \zeta(D-1)\left(1-\delta_{D}^{2}\right)(\mu h)^{2} \\
& +\frac{1}{\pi} a^{\frac{4-D}{2}} h^{-\frac{D+2}{2}} \sum_{n_{1}, n_{2}=1}^{\infty}\left(\frac{n_{2}}{n_{1}}\right)^{\frac{D-2}{2}} K_{\frac{D-2}{2}}\left(\frac{2 \pi a n_{1} n_{2}}{h}\right)(\mu h)^{2},
\end{aligned}
$$

where $\delta_{D}^{2}$ and $\delta_{D}^{3}$ are the Kronecker delta. When $\mu a \gg 1$ and $\mu h \gg 1$, the approximate energy is

$$
E^{D} \sim-3 a^{1-D}\left(\frac{\mu a}{2 \pi}\right)^{\frac{D-1}{2}} e^{-\mu a}-a h^{-D}\left(\frac{\mu h}{2 \pi}\right)^{\frac{D}{2}} e^{-\mu h} .
$$

In the $D=3$ case, Eq.(29) is reduced to

$$
E=E_{\mu=0}+\left[\frac{a}{24 h}+\frac{1}{\pi} a^{\frac{1}{2}} h^{-\frac{1}{2}} \sum_{n_{1}, n_{2}=1}^{\infty}\left(\frac{n_{2}}{n_{1}}\right)^{\frac{1}{2}} K_{\frac{1}{2}}\left(\frac{2 \pi a n_{1} n_{2}}{h}\right)\right] \mu^{2} .
$$


The pressure on the $x^{1}$ direction is

$$
P_{a}=P_{a, \mu=0}+\left[\frac{1}{24 h}-2 a^{\frac{1}{2}} h^{-\frac{3}{2}} \sum_{n_{1}, n_{2}=1}^{\infty} n_{2}^{\frac{3}{2}} n_{1}^{\frac{1}{2}} K_{\frac{1}{2}}\left(\frac{2 \pi a n_{1} n_{2}}{h}\right)\right] \mu^{2} .
$$

By the numerical calculation, we plot $\Delta P_{a}=P_{a}-P_{a, \mu=0}$ in Fig. 3 for $h=4$ and we find $\Delta P_{a}=0$ appears at $a=r_{c r i t}^{\prime} h$, where $r_{c r i t}^{\prime}=0.523522 \cdots$. That is, the pressure is independent of the mass at $r=r_{\text {crit }}^{\prime}$. When $a<r_{\text {crit }}^{\prime} h, \Delta P_{a}<0$ and when $a>r_{c r i t}^{\prime} h, \Delta P_{a}>0$. The larger the mass $\mu$ is, the more deviation is.

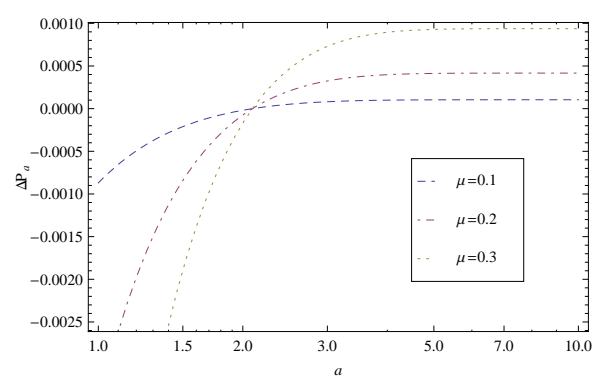

Fig. 3. The difference of the pressure of massive field and massless field on the $x^{1}$ direction $\Delta P_{a}=P_{a}-P_{a, \mu=0}$ for $D=3, h=4$ and $\mu=0.1,0.2,0.3$, respectively. It is clearly seen that $\Delta P_{a}=0$ appears at $a=r_{c r i t}^{\prime} h$, where $r_{c r i t}^{\prime}=0.523522 \cdots$. When $a<r_{c r i t}^{\prime} h, \Delta P_{a}<0$ and when $a>r_{c r i t}^{\prime} h, \Delta P_{a}>0$. The larger the mass $\mu$ is, the more deviation of $\Delta P_{a}$ from $\Delta P_{a}=0$ is.

\section{Conclusions}

In this work we use the Chowla-Selberg formula and its generalization to calculate the Casimir effect with a helix boundary condition in $(D+1)$-dimensional spacetime. We obtain the exact results of the Casimir energy density and pressure for any $D$ for both massless and massive scalar fields.

In this work, we present for the first time (as far as we know) a topology of flat $(D+1)$-dimensional spacetime. This topology causes the helix boundary condition for a Hermitian scalar field. With the new boundary condition, the spectrum of the field will have new feature, which will lead to the observable effect.

The main conclusions of this work are:

- Once topology of spacetime is fixed, the ratio of the sizes of the helix will be a decisive factor. In our case, the negative pressure will become positive with the ratio $r$ of the lengths increasing. This phenomenon of quantum physics is similar to that in the rectangular cavity with Dirichlet condition 89 .

- Both in massless and massive cases, there are critical values $r_{c r i t}(\mu)$. When $a=r_{\text {crit }} h$ or $h=r_{\text {crit }} a$, the pressure $P_{a}$ or $P_{h}$ vanishes. In the $\mu=0$ case, $r_{\text {crit }}(0)=1.52007606 \cdots$ for $D=3$. 
- For a massive field, when the mass $\mu$ tends to zero, the pressure approaches the result of the pressure in massless case and when the mass $\mu \gg 1$, the pressure for a massive field goes to zero. There is another critical value $r_{\text {crit }}^{\prime}$ and the pressure is independent of the mass $\mu$ at $r=r_{\text {crit }}^{\prime}$. In the $D=3$ case, $r_{\text {crit }}^{\prime}=0.523522 \cdots$.

- The summation formulae in the number theory are very useful such as the Chowla-Selberg formula and its generalization for the quantum physics.

Further, the numerical calculation shows that there is a $Z_{2}$ symmetry of $a \leftrightarrow h$. It is not surprised because the boundary conditions (6) and (7) are equivalent to (6) and (8) . Therefore, both $P_{a}$ and $P_{h}$ have the same characteristics.

\section{Acknowledgments}

This work is supported by National Nature Science Foundation of China under Grant Nos.10671128 and 11047138, the Key Project of Chinese Ministry of Education.(No211059), Innovation Program of Shanghai Municipal Education Commission(11zz123), National Education Foundation of China Grant No. 2009312711004 and Shanghai Natural Science Foundation, grant No. 10ZR1422000.

\section{References}

1. M. Bordag, G. L. klimchitskaya, U. Mohideen and V. M. Mostepanenko, Advances in the Casimir Effect, Oxford: Oxford University Press, 2009.

2. C. J. Feng and X. Z. Li, Phys. Lett. B 691, 167(2010).

3. X. H. Zhai, X. Z.Li and C. J. Feng, Mod. Phys. Lett. A 26, 669(2011).

4. J. R. Munkres, Elements of Algebric Topology, Addison-Wesley Publishing Company, Amdterdam, 1984.

5. E. Elizalde, S. D. Odintsov, A. Romeo, A. A. Bytsenko and S. Zerbini, Zeta Regularization Techniques with Applications, World Scientific, Singapore, 1993.

6. X. Z. Li, X. Shi and J. Z. Zhang, Phys. Rev. D 44, 560(1991).

7. I. H. Brevik and E. Elizalde, Phys. Rev. D 49, 5319(1994).

8. X. Z. Li, H. B. Cheng, J. M. Li and X. H. Zhai, Phys. Rev. D 56, 2155 (1997).

9. X. Z. Li and X. H. Zhai, J. Phys. A 34, 11053(2001).

10. S. C. Lim and L. P. Teo, J. Phys. A 40, 11645 (2007).

11. L. P. Teo, Phys. Rev. D 82, 105002 (2010).

12. X. Shi and X. Z. Li, Class. Quant. Grav. 8, 75(1991).

13. R. M. Cavalcanti, Phys. Rev. D 69, 065015(2004).

14. M. P. Hertzberg, R. L. Jaffe, M. Kardar and A. Scardicchio, Phys. Rev. Lett. 95, 250402(2005).

15. X. H. Zhai and X. Z. Li, Phys. Rev. D 76, 047704(2007).

16. X. H. Zhai, Y. Y. Zhang and X. Z. Li, Mod. Phys. Lett. A 24, 393(2009).

17. S. C. Lim and L. P. Teo, Annals Phys. 324, 1676(2009).

18. C. H. Eab, S. C. Lim and L. P. Teo, J. Math. Phys. 48, 082301 (2007).

19. S. C. Lim and L. P. Teo, Phys. Lett. B 679, 130 (2009).

20. E. Elizalde, J. Phys. A 27, 3775 (1994).

21. E. K. Abalo, K. A. Milton and L. Kaplan, Phys. Rev. D 82, 125007 (2010).

22. J. Ambjøn and S. Wolfram, Annals Phys. 147, 1 (1983). 